\title{
Wie steht es um unsere Berufsentwicklung?
}

\section{Michel Matter}

Dr. med., Vizepräsident der FMH, Departementsverantwortlicher Dienstleistungen und Berufsentwicklung

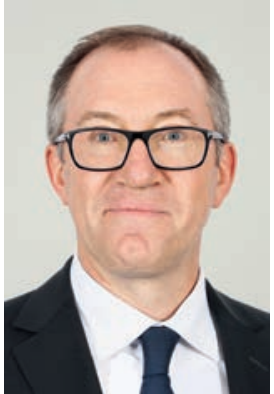

Gerade hat das Parlament beschlossen, dass Ärztinnen und Ärzte, die in der Schweiz eine Praxis eröffnen möchten, drei Jahre in einer anerkannten schweizerischen Weiterbildungsstätte in der für die Zulassung beantragten Fachdisziplin arbeiten müssen. Zudem müssen sie die Sprache der Region sprechen, in der sie praktizieren möchten. Dennoch gibt es mehr Fragen denn je; schliesslich hat das Parlament den Kantonen auch das Recht eingeräumt, die Zahl der Leistungserbringer zu steuern.

Die neue Dreijahresregelung schränkt die Zahl der Weiterbildungsplätze deutlich ein. So stehen für Kardiologen statt wie bisher über 4400 Weiterbildungsstätten zukünftig nur noch 58 zur Verfügung (Gesamtzahl der anerkannten Weiterbildungsstätten).

Offen ist jedoch die Frage nach der Anzahl der Ärztinnen und Ärzte. Wir hören oft, es gebe zu viele Fachärzte, während in der Grundversorgung Mangel bestehe. Seit einigen Jahren wird gefordert, Letztere massiv zu unterstützen. Dennoch bevorzugen die Studierenden der Medizin nach wie vor Facharzt- oder Doppeltitel. Derzeit können sie sich bei der Weiterbildung frei nach den an den verschiedenen Stätten verfügbaren Plätzen richten. Wird dies so bleiben? In der Schweiz gibt es keine Auswahlverfahren oder Zuweisungen, keine Verpflichtung, während der Weiterbildungszeit in einer Randregion zu arbeiten, keine Beschränkung im Hinblick darauf, Teile der Weiterbildung im Ausland zu absolvieren. Auch wenn es immer schwieriger wird, müssen wir weiter dafür kämpfen, dass die Wahl des Fachgebiets eine Herzensentscheidung bleibt. Unser Beruf basiert auf Vertrauen und zwischenmenschlichen Beziehungen, bringt aber auch die Verpflichtung mit sich, sich über das gesamte Berufsleben hinweg weiterzubilden. Wie soll man sich auf Dauer für eine medizinische Tätigkeit begeistern, die nicht den eigenen Wünschen und Träumen entspricht? Wofür hat man dann so lange studiert?

Einige Politikerinnen und Politiker wollen die Anzahl der in einem Kanton tätigen Ärztinnen und Ärzte selbst bestimmen. Rein zufällig entsprechen die vorgeschlagenen Zahlen dem aktuellen Bestand, als wäre dies das Nonplusultra. Angenommen, ein solcher Be- schluss würde - unter dem Vorwand kantonaler Planung oder willkürlich auf Druck der Versicherer - gefasst, welche Auswirkungen hätte dies auf die Zukunft der Studierenden der Medizin, die Assistenzärztinnen und -ärzte sowie die Oberärztinnen und -ärzte, die derzeit in universitären Einrichtungen oder anerkannten Weiterbildungsstätten beschäftigt sind? Werden sie dann gegen die Wand laufen? Werden die Spitäler in der Lage sein, diese hochqualifizierten Ärztinnen und Ärzte auf Dauer zu halten? Werden nach ausländischem Vorbild Positionen für Spitalärzte geschaffen? Wird der Staat in der Lage sein, alle diese Gehälter zu zahlen? Wird er eine Schwemme an Spitalärztinnen und Spitalärzten oder einen unlauteren Wettbewerb vermeiden können? Müssen ältere Ärztinnen und Ärzte auf ihr Recht zu praktizieren verzichten, damit die jüngeren zum Zug kommen? Wenn man sich vor Augen führt, dass die Behörden nach Erreichen des Rentenalters eine Ausübung ärztlicher Tätigkeiten in Universitätsspitälern untersagen, stellt sich die Frage, was die Zukunft für unsere älteren Kolleginnen und Kollegen bringen wird. Wir fördern den immer wieder fruchtbaren Austausch zwischen den Generationen sowie den Erfahrungsaustausch im Hinblick auf den technologischen Wandel und auf die wahnwitzige Idee, die Dauer von Konsultationen vorzugeben.

Die Welt verändert sich, das Medizinstudium ist ebenso wie die paramedizinischen Berufe - attraktiv und beliebt. Ein Teil der Antwort auf die Weiterentwicklung der Patientenversorgung ist Interprofessionalität. Es kann aber nicht sein, dass Kolleginnen und Kollegen keine berufliche Zukunft haben sollen, nur weil bestimmte Regelungen auferlegt werden. Es sollte nicht vergessen werden, dass Spitalärzte oft Kollegen und andere Angehörige des Gesundheitswesens fortbilden. Wer wird diese Aufgabe übernehmen? Die Entscheidungen - und das Erwachen danach - werden brutal sein. Angesichts dieser Aussichten müssen wir uns für eine abgestimmte Planung einsetzen, bei der die Vorstellungen der kantonalen Ärztegesellschaften Gehör finden. Dies ist - zum Wohle aller, aber auch zum Wohle der Qualität in der Gesundheitsversorgung und der Gesundheitskosten - unverzichtbar. 